Progress Report

on Grant \#DE-FG02-92ER75710

\author{
Experimental Eyaluation of an Instrumented Synthesis \\ Method for the Real-Time Estimation of Reactivity
}

1. Scope: This report concerns the first and second quarters supported under this grant. The period covered is 1 July $1992-31$ December 1992.

2. Organization: The research is being directed by Dr. John A. Bernard and Professors David D. Lanning and Allan F. Henry. Two students are active on the project. These are Lt. Lorin Selby and Mr. Weng-Sheng Kuo. Lt. Selby is a nuclear-qualified naval officer and is an M.S./NE degree candidate. Mr. Kuo is a Ph.D. candidate. Both are in the Department of Nuclear Engineering. We expect another student, Mr. Jeff Hughes, to join the group in February 1993.

3. Research Status: Summarized here is the progress that has been made on each task:

a) Theoretical Characterization of Flux Shapes: The objective of this task is to use theoretical methods to determine the spatial shape of the MIT Research Reactor's flux distribution under a variety of skewed shim blade positions. A three-dimensional computer-based nodal model of the MITR-II is being developed. However, the present three-group cross-section set which might be used with that model requires updating, particularly for the effect of fission products. Accordingly, one part of this task is to obtain an accurate two- or three-group cross-section set and then calculate the spatial (3D) flux shapes. The latter calculation will be done using the Monte Carlo code (MCNP). Mr. Weng-Sheng Kuo is performing this work under the direction of Professor Allan F. Henry. Mr. Kuo's efforts are building on those of several other students who have been active in this area of study. These include Mr. Thomas DeLorey, Mr. Everett Redmond, and Mr. Ilkar Tari who have developed MCNP models of the MIT Research Reactor for use in our neutron capture therapy program. The triangle -2 nodal code to be used to find flux shapes for the synthesis method is being developed by Mir. Tom DeLorey.

DISCLAIMER

This report was prepared as an account of work sponsored by an agency of the United States Government. Neither the United States Government nor any agency thereof, nor any of their employees, makes any warranty, express or implied, or assumes any legal liability or responsibility for the accuracy, completeness, or usefulness of any information, apparatus, product, or process disclosed, or represents that its use would not infringe privately owned rights. Reference herein to any specific commercial product, process, or service by trade name, trademark, manufacturer, or otherwise does not necessarily constitute or imply its endorsement, recornmendation, or favorine by the United States Government or any agency thereof. The views and opinions of authors expressed herein do not necessariiy state or reflect those of the United States Government or any agency thereof. 


\section{Sketch of the Theory}

For any theoretical model of transient reactor behavior, a formally exact expression for reactivity can be written symbolically as:

$$
\rho(\mathrm{t})=\frac{\left\langle\phi^{*}|\mathrm{M}(\mathrm{t})-\mathrm{L}(\mathrm{t})| \phi(\mathrm{t})\right\rangle}{\left\langle\phi^{*}|\mathrm{M}(\mathrm{t})| \phi(\mathrm{t})\right\rangle}
$$

where $M(t)$ is a (time-dependent) net fission neutron production operator, $L(t)$ is the net neutron loss operator, $\phi(t)$ is the instantaneous space-energytime dependent flux, and $\phi^{*}$ is a time-independent weight function (usually an adjoint flux corresponding to some reference critical condition).

If the transport equation is used as the theoretical model, $\phi(t)$ will be the directional flux density $\psi(r, \Omega, E, t) ; M(t)$ and $L(t)$ will be integral and integro-differential operators. For the nodal model we propose to use for the present project, if there are $N$ nodes and $G$ energy-groups, $\phi(t)$ will be an NG-element column vector whose elements are the node-averaged group fluxes; and $M(t)$ and $L(t)$ will be (NG) $x(N G)$ matrices involving nodeaveraged group cross sections.

Insertion of the reactivity, along with the prompt neutron lifetime and effective delayed neutron fractions into the point kinetics equations permits calculation of the amplitude function, $T(t)$, defined as:

$$
T(t) \equiv\left\langle\phi *\left|v^{-1}\right| \phi(t)\right\rangle
$$

where, for the nodal model, $y^{-1}$ is an (NG) $x$ (NG) diagonal matrix of inverse group-velocities.

The prompt neutron lifetime and effective delayed neutron fraction for the ith precursor group are defined as:

$$
\Lambda(\mathrm{t}) \equiv \frac{\left\langle\phi^{*}\left|\mathrm{v}^{-1}\right| \phi(\mathrm{t})\right\rangle}{\left\langle\phi^{*}|\mathrm{M}(\mathrm{t})| \phi(\mathrm{t})\right\rangle}
$$

and

$$
\beta_{\mathrm{i}}(\mathrm{t}) \equiv \frac{\left\langle\phi^{*}\left|\mathrm{M}_{\mathrm{di}}(\mathrm{t})\right| \phi(\mathrm{t})\right\rangle}{\left\langle\phi^{*}|\mathrm{M}(\mathrm{t})| \phi(\mathrm{t})\right\rangle}
$$


where $M_{d i}(t)$ is the delayed neutron production operator for the ith precursor group.

If $\rho, \Lambda$ and the $\bar{\beta}_{i}$ are known, $T(t)$ can be determined by solving the point kinetics equations. Conversely, if $\mathrm{T}, \Lambda$ and the $\beta_{\mathrm{i}}$ are known, $\rho(\mathrm{t})$ can be inferred by inserting $T(t)$ into the point equations - a procedure often called "inverse kinetics." Moreover, since the (generally small) variation of $M(t)$ and the $M_{\mathrm{di}}(t)$ with the time usually affects $\Lambda(t)$ and the $\bar{\beta}_{\mathrm{i}}(\mathrm{t})$ to a negligible extent, Equations (2), (3) and (4) imply that knowledge of $\phi(t)$ is all that is needed to infer $\rho(t)$ by inverse kinetics. Specifically, no direct information about control rod motion or feedback effects is required.

The method for determining $\phi(t)$, derived and tested numerically by Jacqmin as described in our original proposal, is based on distributing a set of $\mathrm{J}$ neutron counters at various spatial locations throughout the reactors.

If $<\Sigma_{\mathrm{j}} \mid$ represents an NG row vector of homogenized detector cross sections (all but $\mathrm{G}$ elements of which are zero since the detector is present in only one of the $\mathrm{N}$ nodes), the counting rate $\mathrm{C}_{j}(\mathrm{t})$, for the jth detector is given by:

$$
C_{j}(t)=\left\langle\Sigma_{j} \mid \phi(t)\right\rangle
$$

Then if we approximate the column vector $\phi(t)>$ as a linear combination of $\mathrm{K}$ predetermined time-independent vectors $\mid \phi_{k}>$ so that

$$
\begin{aligned}
& |\phi(t)\rangle=\sum_{k=1}^{K} a_{k}(t)\left|\phi_{k}\right\rangle \\
& C_{j}(t)=\sum_{k=1}^{K} a_{k}(t)<\Sigma_{j}\left|\phi_{k}\right\rangle ; j=1,2 \ldots J
\end{aligned}
$$

These $\mathrm{J}$ equations can be solved by a least squares method for the $a_{k(t)} \mid \phi(t)>$ can be reconstructed from Equation (6); $T(t), \rho(t)$ and the $\beta_{i}(t)$ can be computed from Equations (2), (3), and (4), and $\rho(t)$ can be determined from the point kinetics equations.

The theoretical analysis thus requires computation of static nodal flux shapes which when blended together with time-dependent coefficients will provide a good approximation to $|\phi(t)\rangle$ throughout the transient being analyzed. To find these along with the node-averaged counter cross sections $<\Sigma_{\mathrm{j}}$ l, we need a three-dimensional nodal code along with some 
way of determining equivalent, node-homogenized, group cross sections for that code. We have made progress in both these areas.

\section{The Nodal Code}

A radial slice of the MIT Reactor is shown in Figure One. The fuel elements are composed of highly enriched uranium-aluminum alloy plates $-6.5 \mathrm{~cm}$ wide, cooled with $\mathrm{H}_{2} \mathrm{O}$. Outside the cylindrical tank is a $\mathrm{D}_{2} \mathrm{O}$ reflector followed by graphite (not shown). The core region is best described in triangle- $Z$ geometry, and we are constructing a few-group, triangle- $\mathrm{Z}$ nodal code for this purpose. In order to improve the accuracy of the nodal equations we use what is called a "quadratic" nodal model. Thus, (referring to Figure Two) we define a "transverse-averaged" flux $\bar{\phi}_{\mathrm{gx}}(\mathrm{x})$ by:

$$
\bar{\phi}_{g x}(x) \equiv \frac{\int_{-\frac{y(x)}{2}}^{\frac{y(x)}{2}} \phi(x, y) d y}{\int_{-\frac{y(x)}{2}}^{\frac{y(x)}{2}} d y}
$$

and approximate it by the quadratic

$$
\bar{\phi}_{\mathrm{gx}}(\varsigma)=\left(1-3 \zeta+2 \varsigma^{2}\right) \phi_{\mathrm{g}}(0,0)-\left(3 \varsigma-4 \varsigma^{2}\right) \bar{\phi}_{\mathrm{gx}}(\mathrm{d})+\left(6 \varsigma-6 \varsigma^{2}\right) \bar{\phi}_{\mathrm{g}}
$$

where $\bar{\phi}$ is the volume-averaged nodal flux and

$$
\left(\varsigma \equiv \frac{x}{d}\right)
$$

If the net current $\bar{J}_{g x}(d)$ is related to $\bar{\phi}_{d x}(d)$ by Fick's Law and triangles extending from $x=-d$ to $x=3 d$ are included, a considerable amount of algebraic manipulation leads to the elimination of the point flux $\phi_{g}(0,0)$ and the face-averaged flux $\bar{\phi}_{g x}(d)$ and results in an equation relating $\bar{J}_{g x}(d)$, $\overline{\mathrm{J}}_{\mathrm{gx}}(-\mathrm{d})$, and $\overline{\mathrm{J}}_{\mathrm{gx}}(3 \mathrm{~d})$ to the volume-averaged flux $\bar{\phi}_{\mathrm{g}}$ and those belonging to one triangle on the left and two triangles on the right. Repeating this procedure for directions perpendicular to the other two faces of the triangle and making use of the nodal balance equation for the triangle yields a set of 
equations which can be solved for the $\bar{\phi}_{\mathrm{g}}$ 's and thus the $\left(\phi_{\mathrm{k}}>\right.$ 's of Equation (6).

The equations relating the $\overline{\mathrm{J}}_{\mathrm{gx}}$ 's to the $\bar{\phi}_{\mathrm{g}}$ 's are approximate, since they are based on Fick's Law, node-homogenized, few-group cross sections and the quadratic approximation (Equation (9)). To correct for this, discontinuity factors have been introduced so that, if a reference solution is known and used to compute these correction factors, the node-averaged group-fluxes found by solving the nodal equations will agree exactly with those edited from the reference calculation.

Another set of discontinuity factors has been introduced to speed the solution of the nodal equations. This second set corrects the finitedifference expression for the $\bar{J}_{g x}\left(d^{\prime} s\right)$ so that it yields the quadratic result. The factors that do this are computed iteratively as the calculation proceeds. The global equations that result have the same structure as the standard, mesh-centered, finite difference equations and, in fact, reduce exactly to these equations if both sets of discontinuity factors are set to unity. Since the global nodal equations have the finite difference form, it is possible to determine the first (fixed) set of discontinuity factors so that they force the finite difference equations to match the reference calculation. However, with only two triangles per fuel assembly, this procedure can lead to significant errors if any approximate reference calculation is performed (for example, one involving only a single fuel assembly rather than a full core calculation). In view of this situation, we feel that the quadratic approximation is more efficient.

A static version of the quadratic, nodal, triangle- $Z$ code has been programmed and tested by Tom DeLorey. For very small mesh spacings the nodal difference equations have been found to be unstable for certain sets of boundary conditions. However, the mesh spacings of interest for the present application are well in the range of stability.

A time-dependent extension of the code is now being programmed. It will provide the capability of analyzing entirely theoretically the transients to be run in the future stages of the project.

\section{Determination of Few-Group Cross Sections}

The nodal code requires as input homogenized few-group parameters and discontinuity factors to correct for spectrum, transport, and heterogeneity effects. We are attempting to find these parameters by Monte Carlo methods. The Los Alamos code MCNP is being used for this purpose. 
As a first step in developing a Monte Carlo scheme for finding nodal parameters, we have concentrated on fuel element Al of Figure One. Fortunately, for a different project, Everett Redmond, an MIT graduate student, has run a full, three-dimensional MCNP problem for the MIT Reactor. Figure Three shows a radial slice of the specific geometrical configuration analyzed. (The reflector, not shown on the figure, was included in the calculation.) Three-group, homogenized cross sections for fuel element $\mathrm{A} 1$ have been edited from the result for the following group structure:

$$
\begin{array}{ll}
\text { Group 1: } & 0-0.4 \mathrm{ev} \\
\text { Group 2: } & 0.4-3 \mathrm{kev} \\
\text { Group 3: } & 3 \mathrm{kev}-10 \mathrm{mev} .
\end{array}
$$

Results for the absorption cross section versus axial position are shown in Figure Four.

Statistical fluctuations are evident for the thermal and intermediate group.

To decrease these fluctuations as well as to decrease (to a very major extent) the computational cost for the MCNP calculation, fuel assembly A1 was isolated and analyzed by MCNP, subject to zero net current boundary conditions. This work was carried out by Ilkar Tari. Figure Five shows the absorption cross section versus axial position for this case. The statistical fluctuations are greatly reduced. Moreover, it appears that smoothing the curves on Figure Four would yield results quite similar to those of Figure Five.

We are encouraged by these results. However, much work remains to be done. We have not yet used MCNP to determine the face-averaged currents needed to compute discontinuity factors. Also, we have not yet attempted to find node-homogenized cross sections for the detectors which will be located in three of the one-inch-diameter water holes adjacent to and opposite from the regulator rod or for the nodes containing control blades. (See Figure One.) Finally, we must investigate whether isolated assembly calculations can provide acceptably accurate nodal parameters for the other assemblies in the core.

b) Experimental Determination of Elux Shapes: This task entails two subtasks:

(i) Installation and Calibration of In-Core Sensons

The core of the MIT Research Reactor is hexagonal in shape. An open tube, suitable for holding small fission chambers, is located at 
each corner of the hexagon (See Figure One). One of these contains the reactor's regulating rod: the others are vacant. Three of these will be used to provide a symmetric pattern for the experiment with three detectors placed in each hole in order to provide information on the axial flux shape. Lt. Selby is responsible for this task and his report follows.

The first item accomplished during their reporting period was the determination of the maximum allowable size for the fission chamber detectors to be used in measuring the flux shape. The best location for placing these detectors is in the water vent holes at the periphery of the core as shown in Figure One. Because these vent holes are designed to allow the passage of displaced water when shim blades are dropped, there was concern that blocking these holes could hinder biade drop times. In order to test this concern, three $1 / 2$ " aluminum rods were obtained to simulate the detectors.

In July 1992 the water vent hole dimensional measurement and shim blade drop time tests were conducted. In the first portion of the test, the $1 / 2$ " aluminum blanks were inserted into each of the water vent holes. They fit into every vent hole except $\# 2$. Hence, because only three water vent holes are required for the research and in order to gain symmetry, water vent holes $\# 1, \# 3$ and $\# 5$ were selected. In the next portion of the test, the $1 / 2$ " aluminum rod was placed in each water vent hole (except $\# 2$ ), the two adjacent shim blades were sequentially raised, and a drop time test was performed. This sequence was repeated for each vent hole. The results of these tests are recorded in Table One. As can be seen from this table, the drop times are all well within the required one second specification. The longer drop times associated with blades \#1 and \#5 are due to the presence of the regulating rod in water vent hole $\# 1$.

In the final portion of this experiment, a $1 / 2$ " aluminum rod was placed in water vent holes $\# 1, \# 3$ and $\# 5$. With the three rods in this configuration, the shim blade drop time test was performed on each blade. The purpose of this test was to ensure that the drop times would still be within specification with three water vent holes partially blocked simultaneously. The results of this test are given in Table Two. As before, all times are well within the required specification.

The aluminum tubes used in the above tests were one-half inch in diameter. Hence, the next step in the project was to approach each of the fission chamber detector vendors and obtain price quotations 
for chambers of that outside diameter. We also specified that each detector operate in the current mode with a minimum sensitivity of $1 \times 10^{-18} \mathrm{Amps} / \mathrm{nv}$ (thermal). The detector vendor best able to meet these specifications with the most competitive price was Imaging and Sensing Technology. The detector selected was the WL-23798. The maximum outside diameter of this detector is 0.188 " and it has a minimum neutron sensitivity of $1 \times 10^{-17} \mathrm{Amps} / \mathrm{nv}$ (thermal). Three detectors were ordered and subsequently received in late December 1992.

With the fission chamber detectors on order, the next priority was the design of the data acquisition system. Because the current from the detectors is expected to be on the order of 1 microamp it was determined that the most effective form of measurement would be with a picoammeter. Three Keithley model 485 s were selected for this function. In order to monitor the signals from each of the three fission chamber detectors, a Data Translation model DT 2801 A/D board was obtained. Each of the Keithley model 485 picoammeters provides a 0-2 volt analog output signal which can be wired to the AND board. A data acquisition software package, Global Lab, will be used to configure the AND board for each data run and for signal conditioning and analysis. The power supply for each of the three fission chamber detectors is a DC floating power supply which was made by wiring several smaller batteries together to obtain the required voltage. 
Table One

Shim Blade Drop Times: Water Vent Holes Open

\begin{tabular}{|c|c|c|}
\hline $\begin{array}{c}\text { Blocked } \\
\text { Water } \\
\text { Vent Hole }\end{array}$ & $\begin{array}{c}\text { Shim Blade } \\
\text { Tested }\end{array}$ & $\begin{array}{c}\text { Drop Time } \\
\text { (ms) }\end{array}$ \\
\hline$\# 1$ & Blade \#1 & $602 / 589^{*}$ \\
\hline$\# 1$ & Blade \#2 & 519 \\
\hline$\# 3$ & Blade \#3 & 481 \\
\hline$\# 3$ & Blade \#4 & 469 \\
\hline$\# 4$ & Blade \#4 & 468 \\
\hline$\# 4$ & Blade \#5 & 486 \\
\hline$\# 5$ & Blade \#5 & 479 \\
\hline$\# 5$ & Blade \#6 & 504 \\
\hline
\end{tabular}

( ${ }^{*}$ Test repeated to verify timing)

Table Two

Shim Blade Drop Times: Warer Vent Holes \#1, \#3, and \#5 Blocked

\begin{tabular}{|c|c|}
\hline $\begin{array}{c}\text { Shim Blade } \\
\text { Tested }\end{array}$ & $\begin{array}{c}\text { Drop Time } \\
\text { (ms) }\end{array}$ \\
\hline Blade \#1 & 568 \\
\hline Blade \#2 & 554 \\
\hline Blade \#3 & 480 \\
\hline Blade \#4 & 474 \\
\hline Blade \#5 & 494 \\
\hline Blade \#6 & 503 \\
\hline
\end{tabular}


The final requirement for the test equipment was the design and construction of a test stand. It was decided that the easiest way to accomplish this was to buy a $1 / 2$ " outside diameter aluminum tube and to put a cap on the lower end. Three fifteen foot aluminum tubes were manufactured and aluminum end caps were machined and welded to the bottom of each tube. The three tubes will be placed in the three selected water vent holes and the detectors will be lowered into each tube. This will keep the detectors and cabling dry, thereby minimizing contamination problems.

Initial testing and out of core calibration of the test equipment are now in progress. Once this is completed the three detectors will be installed in the MITR II core tank in-core flux measurements can be taken. This item is scheduled to be completed in February 1993.

\section{(ii) In-Core Flux Measurements:}

Once the instruments are installed and calibrated, measurements will be made of the reactor power distribution first under normal conditions and then under abnormal conditions by moving the blades so that they are no longer in the normal banked position.

Lt. Selby is also responsible for this task. The MIT Research Reactor is currently running continuously ( $24 \mathrm{hrs} /$ day, seven days/week) in support of several other experiments. That run is schedule to end in mid-February. Accordingly, we anticipate the start of in-core flux measurements for this project in late February 1993. These measurements will be conducted at powers of less than $1 \mathrm{~kW}$ because of a limitation in the MITR-II's technical specifications that precludes operation with a skewed shim blade bank at appreciable power levels.

c) Experimental Evaluation of Instrumented Synthesis Method: This task is scheduled to start in March 1993. The results of tasks \#a and \#b would be compared in order to provide an assessment of the instrumented synthesis method under abnormal conditions by moving the blades so that they are no longer in the normal banked position.

d) Controller Design: This task is scheduled to start in July 1993. Information obtained from the instrumented synthesis method on in-core conditions will be used to construct a digital controller. The objective here is to demonstrate the feasibility of controlling a nuclear reactor via estimation of internal parameters. For example, operation of the MIT Research Reactor is currently limited to those combinations of coolant temperature, coolant flow, and power for which neither incipient boiling nor flow instabilities 
will occur. Currently, correlations that relate ex-core measurements to the onset of these conditions are used to avoid unsafe operation. A more direct and less restrictive approach would be to calculate margins to incipient boiling and flow instabilities from the in-core power distribution. This will be done in order to demonstrate the potential benefits of the instrumented synthesis method.

\section{Problems: None}

5. Developments: We recently issued our sixth report on reactor controls research. It is entitled, "Studies on the Closed-Loop Digital Control of Multi-Modular Reactors." A copy was provided to you earlier (12/22/92). Papers based on that report will be presented at two upcoming conferences. These are the American Nuclear Society's conference on "Nuclear Plant Instrumentation, Control and ManMachine Interface Technologies" to be held at Oak Ridge National Laboratory (ORNL.) in April 1993 and the American Control Conference to be held in San Francisco in June 1993. Enclosed are copies of two recent publications. These are:

a) "Non-Linear Controi of Neutronic Power in Reactors Described by SpaceIndependent Kinetics" which appeared in the September 1992 issue of the Intermational Joumal Control-Theory and Advanced Technology (C-TAT), Vol. 8, No. 3, pp. 495-511.

b) "Issues Regarding the Design and Acceptance of Intelligent Support Systems for Reactor Operators" which appeared in the October 1992 issue of IEEE Nuclear Science, Vol. NS-39, No. 5, pp. 1549-1558. 


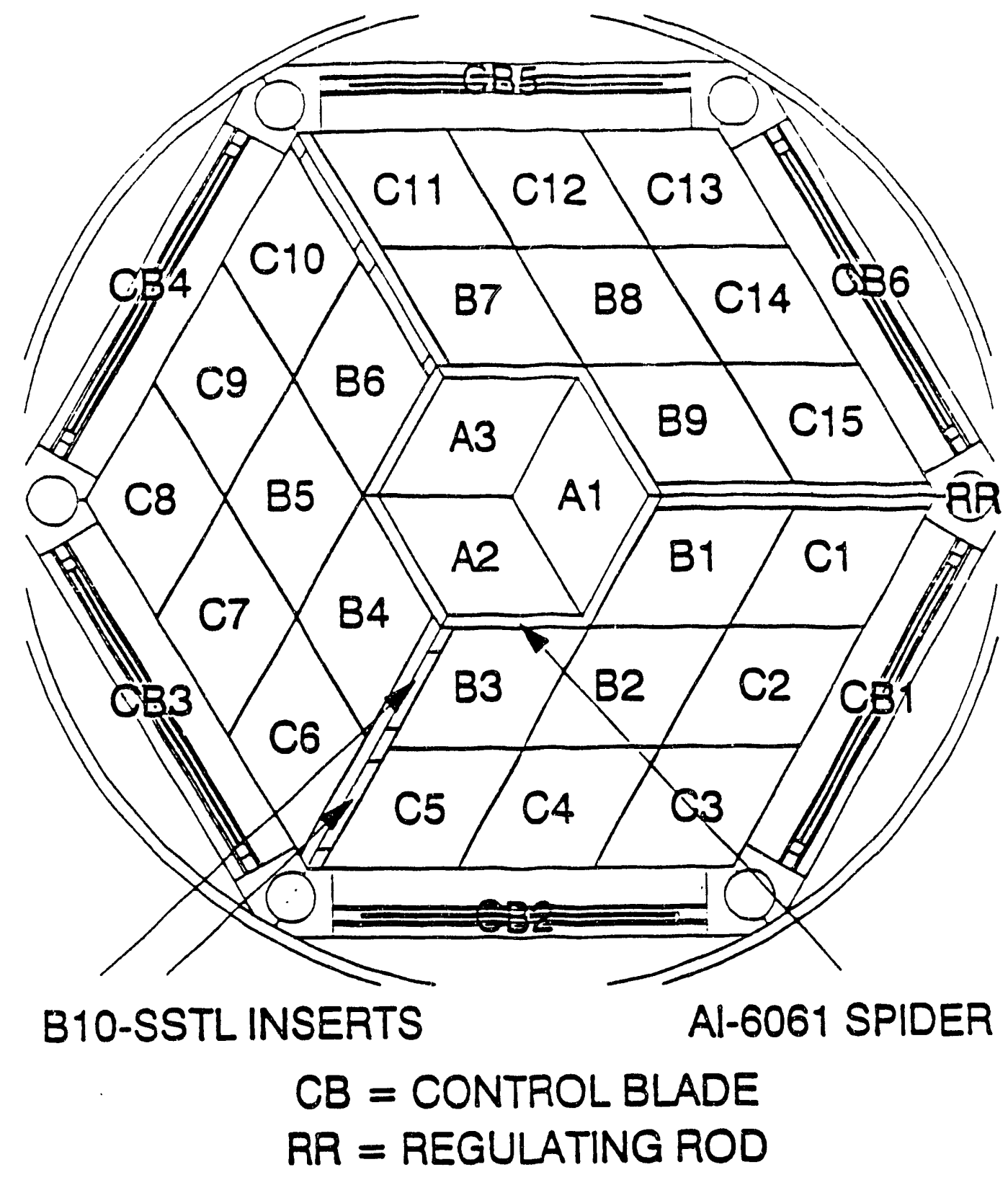

Figure 1 MITR Core Layout

A-2, A-3, B-3, B-6 and B-9 are Al-6160 dummy elements 

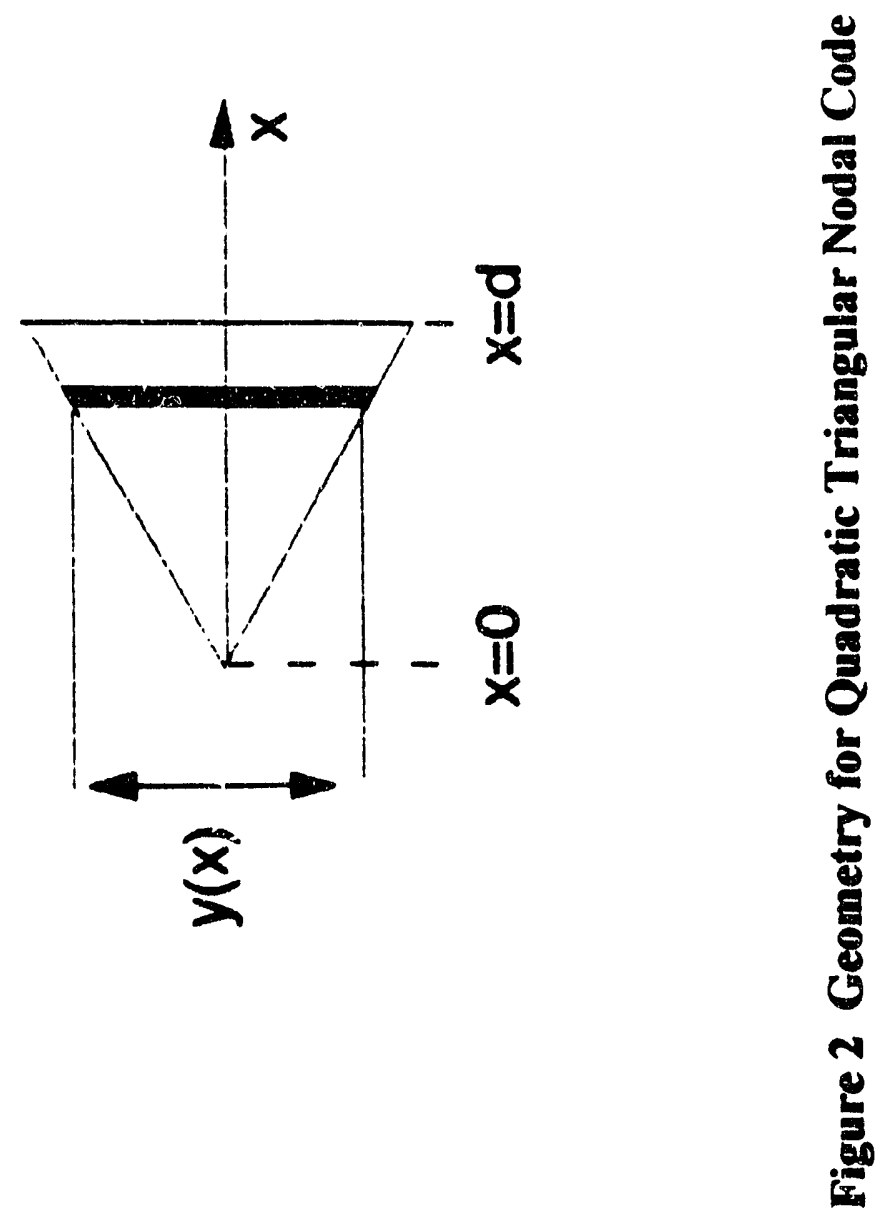


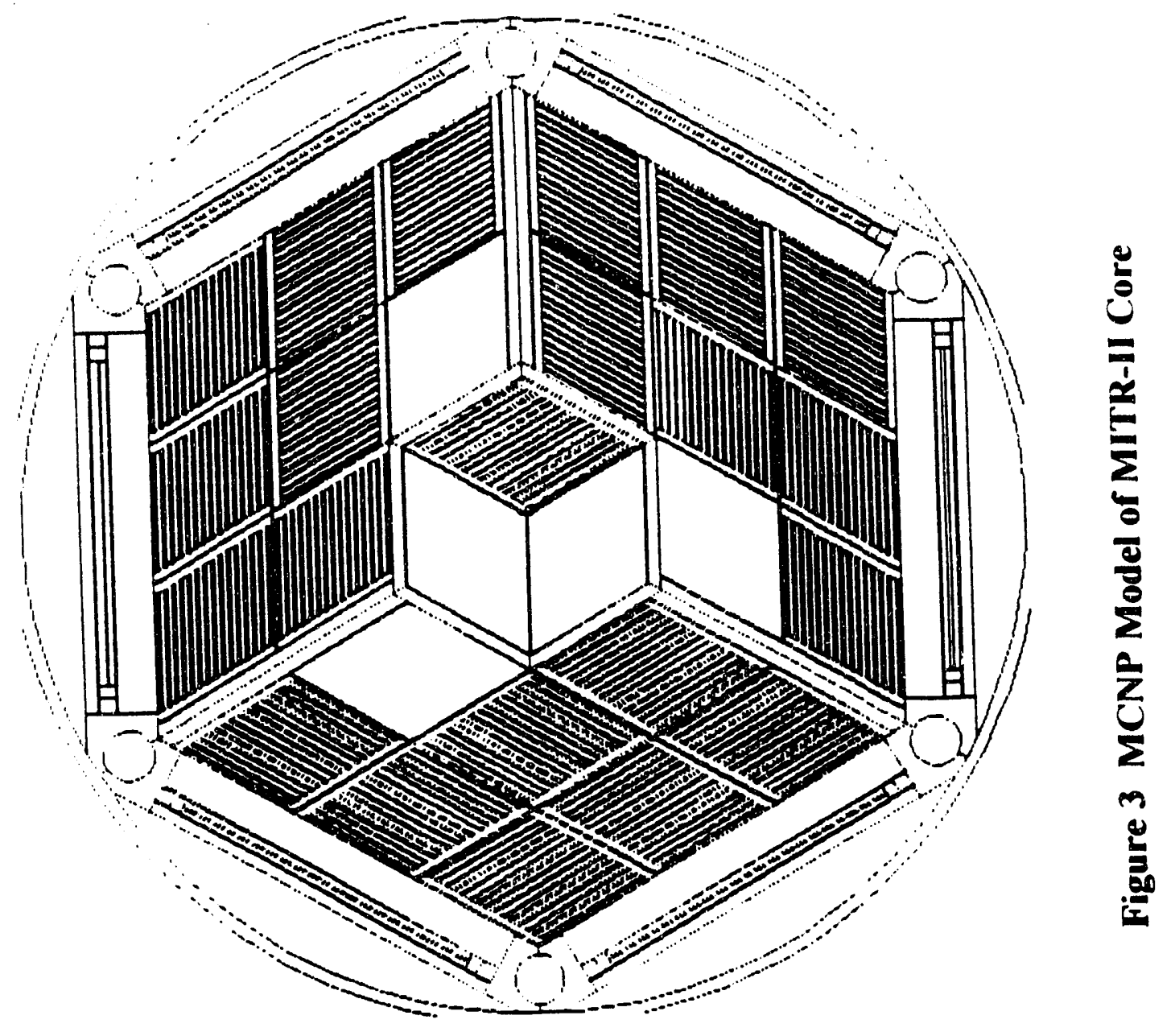




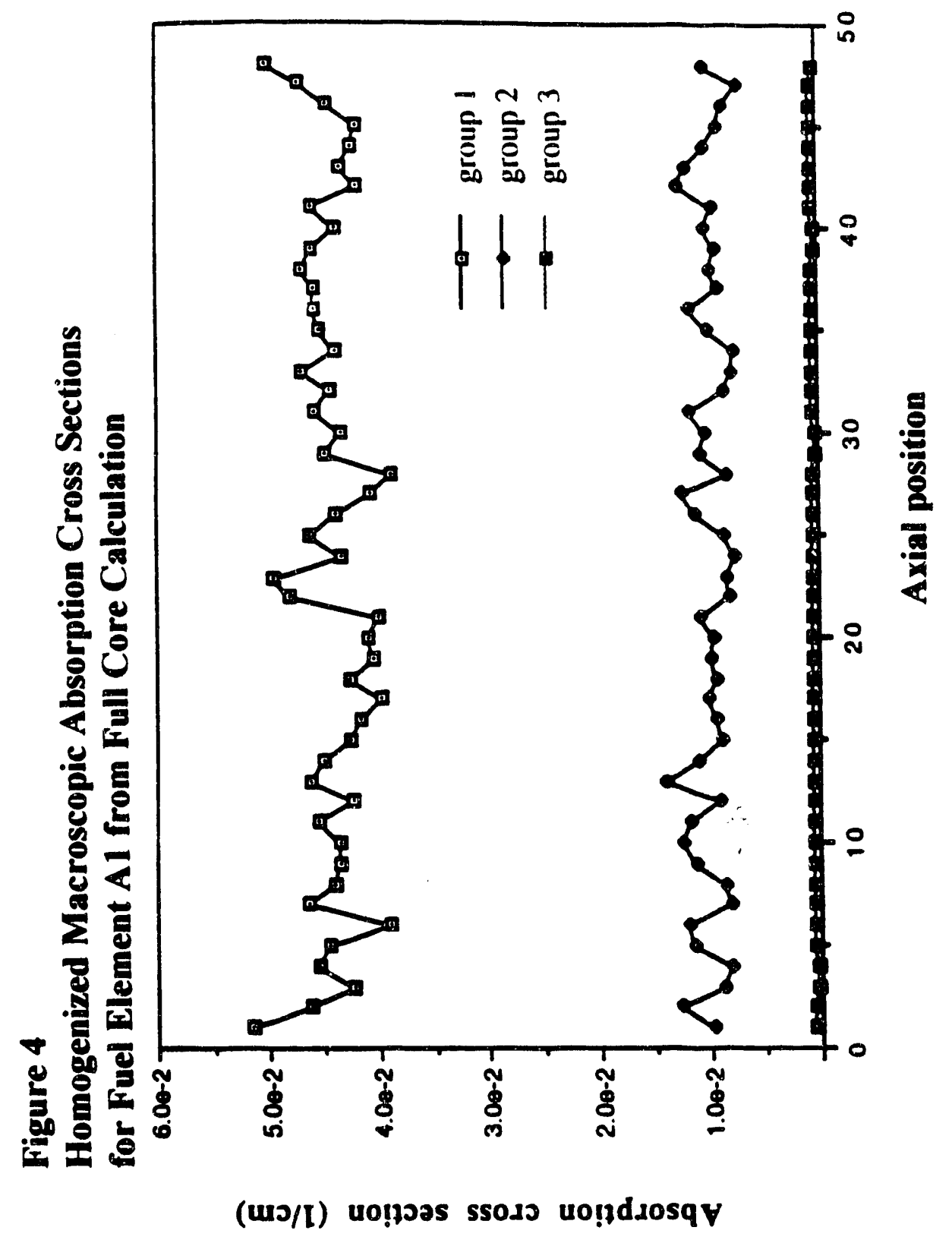




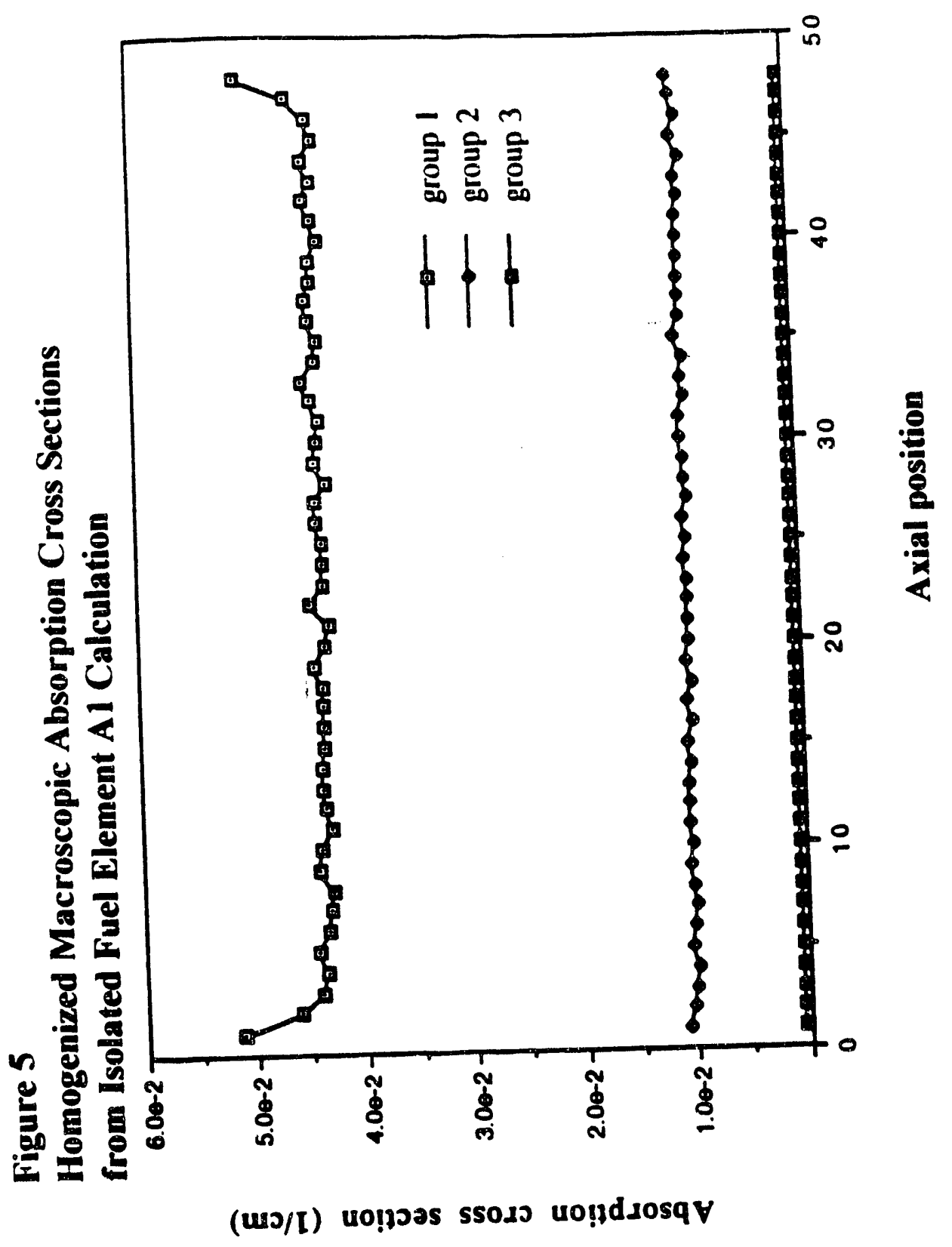



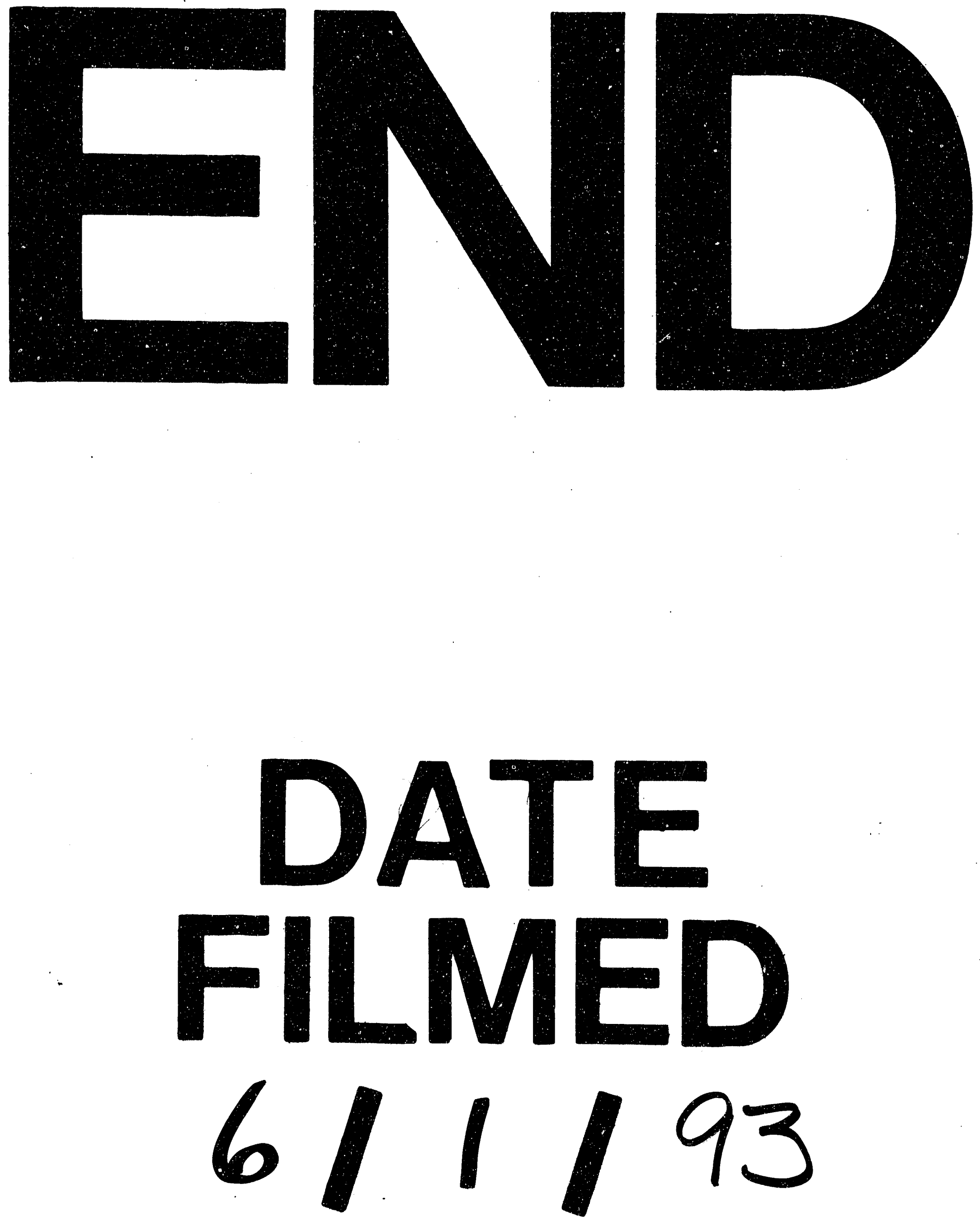
